
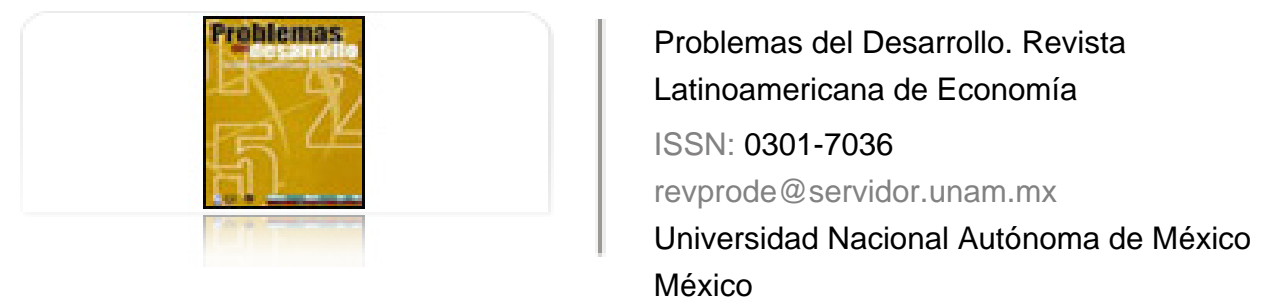

Sales Rocha, Francisco José; Oliveira Vergolino, José Raimundo Políticas públicas en la reducción de la desigualdad del ingreso y de la pobreza: nordeste de Brasil Problemas del Desarrollo. Revista Latinoamericana de Economía, vol. 35, núm. 137, 2004, pp. 161183

Universidad Nacional Autónoma de México

Distrito Federal, México

Disponible en: http://www.redalyc.org/articulo.oa?id=11825947005

Cómo citar el artículo

- Número completo

- Más información del artículo

Página de la revista en redalyc.org

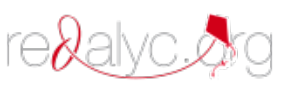

Sistema de Información Científica

Red de Revistas Científicas de América Latina, el Caribe, España y Portugal Proyecto académico sin fines de lucro, desarrollado bajo la iniciativa de acceso abierto 


\title{
Políticas PúBlicas en LA REDUCCIÓN DE LA DESIGUALDAD DEL INGRESO Y DE LA POBREZA: NORDESTE DE BRASIL
}

\author{
Francisco José Sales Rocha* \\ José Raimundo Oliveira Vergolino**
}

Fecha de recepción: 20 de junio de 2002. Fecha de aceptación: 18 de mayo de 2004.

\begin{abstract}
Resumen:
En este trabajo se analizó, empíricamente, el papel de las variables de políticas públicas en la reducción de las desigualdades de renta per capita entre las microrregiones del Nordeste de Brasil, en el periodo de 1970 a 1998. Dicho análisis se realizó con base en la metodología de la descomposición del diferencial de renta y del índice de concentración de renta de Gini. El primero mostró que la parte de la diferencia del producto per capita explicada por la variable de capital humano fue de $32 \%$ para la diferencia entre las microrregiones de la región metropolitana y las del Litoral. El índice de Gini mostró que si no hubiese diferencias significativas en las variables de capital humano - geográfica e institucional entre las microrregiones del nordeste—, la concentración de renta entre éstas sería 37\% menor.
\end{abstract}

Palabras clave: desigualdad, concentración de renta, microrregiones, nordeste de Brasil.

\begin{abstract}
This study makes an empirical analysis of the role of public policy variables in the reduction of income inequalities per capita among the micro-regions of Northeastern Brazil, in the period 1970 to 1998. The empirical analysis was based on an income differential breakdown methodology and the Gini income coefficient. The first showed that part of the differential in the per capita product occurred because the human capital variable was $32 \%$ of the difference between micro-regions in the Metropolitan and Coastal regions. The Gini coefficient showed that there were no significant differences in the human capital variables -i.e. geographical and institutional between the micro-regions of the Northeast - and the concentration of income among these micro-regions would be $37 \%$ less.
\end{abstract}

Key terms: inequality, income concentration, micro-regions, northeastern Brazil, public policies.

* Profesor adjunto de la Universidad Federal de Ceará, Universidad Federal de Pernambuco, Recife, Brasil.

** Profesor adjunto de la Universidad Federal de Pernambuco. 


\section{Résumé}

Dans ce travail, on a analysé de façon empirique le rôle des variables des politiques publiques dans la réduction des inégalités de revenu per capita entre les micro régions du Nord Ouest du Brésil, dans la période de 1970 à 1998. L'analyse empirique a été réalisée grâce à la méthodologie de la décomposition du différentiel de revenu et de l'indice de concentration de revenu de Gini. Le premier a montré que la partie de la différence du produit par capita expliquée par la variable du capital humain a été de $32 \%$ en ce qui concerne la différence entre les micro régions de la région métropolitaine et celles du Littoral. L'indice de Gini a montré que, s'il n'y avait pas de différence significative dans les variables de capital humain - géographique et institutionnelle entre les micro régions du Nord Ouest-, la concentration du revenu entre ces micro régions serait inférieure de $37 \%$.

Mots clés: inégalité, concentration du revenu, micro régions, Nord Ouest, Brésil, politiques publiques.

\section{Resumo}

Este trabalho analisa, empiricamente, o papel das variáveis de políticas públicas na redução das desigualdades de renda per capita nas microrregiões do Nordeste do Brasil, no período de 1970 a 1998. A análise empírica foi realizada com base na metodologia da decomposição do diferencial de renda, com posterior utilização do índice de concentração de renda de Gini. A primeira análise demonstrou que a diferença do produto per capita, explicitada pela variável de capital humano, foi de $32 \%$ entre as microrregiões da região metropolitana e as do Litoral. $O$ índice de Gini mostrou que se não houvesse diferenças significativas nas variáveis de capital humano - geográficas e institucionais nas microrregiões do Nordeste- a concentração de renda entre estas microrregiões seria $37 \%$ menor.

Palavras-chave: desigualdade, concentração da renda, microrregiões, Nordeste, Brasil, políticas públicas. 


\section{Introducción}

$\mathrm{E}$

studios que tratan del proceso de crecimiento económico del nordeste brasileño en los últimos treinta años —como Gustavo Gomes y José Vergolino (1995),

José Vergolino y Aristides Monteiro Neto (1996) — muestran que la actividad productiva y la dinámica de esta región se concentró, básicamente, en las zonas metropolitanas de Salvador, Recife y Fortaleza, debido a la participación de éstas en el producto de la región. En contraste, la Zona de la Mata presentó un grave estancamiento, y el Semiárido señales inequívocas de decadencia. Por otro lado, los polos de irrigación demostraron ser una excelente alternativa de dinamización de la actividad productiva en el nordeste. De esa forma, se concluye que - dada la concentración productiva y la diferencia de dinámica económica entre las subregiones — la desigualdad de renta per capita constituye una señal negativa (Semi-árido muy pobre y con daños sociales significativos) del proceso de crecimiento económico de dicho territorio en los últimos treinta años.

Trabajos recientes, basados en la metodología de la descomposición del diferencial de renta y en el índice de Gini — como Fábio Torres y Jairo Méndez (2000), Rolando Morales, Ervin Gallopo, Luis Jemio, Maria Choque y Natacha Morales (2000), Gerardo Esquivel (2000) y Carlos Azzoni, Naércio Menezes, Tatiane Menezes y Raul Silveira Neto (2000)_, han resaltado la influencia de las variables geográficas (distancia a los principales mercados de un país o región, altitud, latitud, entre otros), de infraestructura (disponibilidad de energía eléctrica), de capital humano (tasa de matrícula en la enseñanza fundamental y media) e institucionales (transferencias municipales de recursos, mortalidad infantil) en las desigualdades de renta per capita entre unidades geográficas.

Los principales resultados obtenidos por estudios que utilizan la metodología de la descomposición del diferencial de renta (o producto per capita) y el índice de concentración de renta de Gini para analizar empíricamente las desigualdades regionales de renta en el país, son los siguientes: Fábio Torres y Jairo Méndez (2000:45-59). En un estudio realizado para los municipios de Colombia, muestran que la diferencia del promedio de renta per capita entre el conjunto de municipios que forman la región andina (área relativamente rica) y el conjunto que forman la del Caribe (área pobre, en 1995) se debe en $82 \%$ a la variable geográfica y $5 \%$ a la de capital humano; y con respeto a la concentración de renta en los municipios colombianos, medida por el índice de Gini, en 1995, este trabajo muestra que la variable geográfica tiene poco efecto sobre la baja de la concentración de renta municipal. Entre tanto, la variable de capital humano posee un efecto significativo: si no hubiese diferencias notables en ésta entre los municipios colombianos, la concentración de renta sería $36 \%$ menor. 
Gerardo Esquivel (2000:39-45), analizando los estados de México, concluye que la diferencia del promedio de renta per capita entre el conjunto de estados del norte (región relativamente rica) y el de los estados del sur (región pobre, en 1995) se debe en 53\% a la variable geográfica y en 36\% a la de capital humano; y con respeto a la concentración de renta en los estados de México, en 1995, medida por el índice de Gini, se verificó que entre $45 \%$ y $63 \%$ de la desigualdad regional de renta se debe a las características geográficas de dichos estados.

Carlos Azzoni et al. (2000:15-20), en un análisis de los estados de Brasil, muestran que su desigualdad de renta -medida por el índice de concentración de renta de Gini, en 1996 - sería $15 \%$ menor si no hubiese diferencias importantes entre ellos en la variable geográfica, y $6.4 \%$ menor si fuesen menos dispares con respeto a la disponibilidad de capital humano.

En síntesis, la idea fundamental de estos estudios es la siguiente: niveles diferentes de disponibilidad — en términos cuantitativos y cualitativos - de variables geográficas, infraestructura, capital humano e institucional pueden afectar la rentabilidad del capital y, consecuentemente, la realización de inversiones en unidades geográficas, resultando en áreas ricas (que atraen niveles altos de inversión y crecen más) y áreas pobres (que atraen niveles bajos de inversión y crecen menos). Así, la movilidad imperfecta de los factores de produción puede crear, o agravar, las desigualdades de renta per capita entre países, regiones, estados o microrregiones. Sin embargo, estas desigualdades pueden reducirse mediante políticas públicas dirigidas, por ejemplo, a la mejoría del capital humano y de la infraestructura.

El objetivo de ese trabajo es analizar los siguientes temas relacionados con la desigualdad de renta per capita entre las microrregiones y, consecuentemente, entre los subconjuntos de las microrregiones de la región metropolitana (MRM), de las microrregiones del Litoral (MLIT), de las microrregiones del Semi-árido (MSA), de las microrregiones del Cerrado (MCER) y de las microrregiones Pre-amazónicas (MPRM) del nordeste del Brasil, en el periodo de 1970 a 1998: a) Cuál fue el efecto de las variables geográfica, de capital humano e institucional sobre el nivel del produto per capita de las microrregiones del nordeste en 1998; $b$ ) Cuál es la participación de las variables geográfica, de capital humano e institucional ante la magnitud de la diferencia del promedio de renta per capita entre los subconjuntos de las microrregiones; c) Cómo las variables geográfica, de capital humano e institucional afectan en forma aislada y en conjunto la concentración de renta entre las microrregiones del nordeste.

El trabajo está dividido en cinco secciones. En la segunda se presentan los aspectos de la teoría del crecimento relacionados con la diferencia de renta per capita entre unidades geográfica, como países, estados y regiones. En la tercera se analiza la concentración de la producción y de la desigualdad de renta per capita entre microrregiones del nordeste 
brasileño. Enseguida se exponen los datos y modelos empíricos utilizados en el estudio. En la quinta se muestran los principales resultados empíricos encontrados a partir de la metodología de la decomposición del diferencial de renta y del índice de Gini. A título de conclusión se resumen las principales contribuciones del trabajo para un mejor entendimiento del problema de la desigualdad de renta per capita entre las microrregiones del nordeste del Brasil.

\section{Bases teóricas}

Según David Romer (1996:6-7), las cuestiones centrales que los modelos de crecimento económico (neoclásicos o no) explican son la diferencia temporal de renta per capita en el nivel de economías entendidas como un todo, es decir, mundo, país, región, estado; y la diferencia espacial de renta per capita entre unidades geográficas. En el presente estudio analizamos la diferencia espacial de renta.

El modelo neoclásico de crecimiento, al utilizar una concepción limitada de capital, no consigue explicar de manera satisfactoria el porqué de las grandes diferencias observadas en el producto por trabajador, entre unidades geográficas desarrolladas y en desarrollo (emergentes). En esta sección se analiza la cuestión de la desigualdad espacial de renta tomando como base que el capital es una variable fundamental para explicar el nivel y la tasa de crecimiento del producto. Para simplificar, se trabaja con el modelo de capital humano propuesto por Gegory Mankiw, David Romer y David Weil (1992:4-16), pues presenta una concepción amplia de capital en su estructura formal y analítica. En este modelo, el logaritmo natural del producto por unidad de trabajo efectivo en el equilibrio de largo plazo se representa por:

$$
\ln y^{*}=\frac{\alpha}{1-(\alpha+\beta)} \ln s_{k}+\frac{\beta}{1-(\alpha+\beta)} \ln s_{H}-\frac{(\alpha+\beta)}{1-(\alpha+\beta)} \ln (n+g)
$$

donde:

sk es el ahorro en capital físico;

sH es el ahorro en capital humano; y

$\mathrm{n}+\mathrm{g}$ es la tasa de crecimiento efectiva del trabajo.

Si $\beta=0$, se obtiene la expresión análoga a la ecuación (2.1) mediante el modelo de crecimiento neoclásico:

$$
\ln y_{\text {Solow }}^{*}=\frac{\alpha}{1-\alpha} \ln s_{k}-\frac{\alpha}{1-\alpha} \ln (n+g)
$$


Para analizar la utilidad del modelo de capital humano para explicar la desigualdad de renta per capita entre unidades geográficas, es necesario determinar una estimativa de (participación del capital humano en la renta). David Romer (1996:134) considera el siguiente intervalo para $\beta$ : $\frac{(1-\alpha)}{2}<\beta<\frac{2(1-\alpha)}{3}$, asumiendo $\alpha=1 / 3 \Rightarrow 1 / 3<\beta<4 / 9$.

La importancia del capital humano puede constatarse en la siguiente forma:

Admitiendo que $\alpha=0,35$ e $\beta=0,40$, y substituyendo estos valores en las ecuaciones (2.1) y (2.2):

a) En la ecuación (2.1):

$\ln y^{*}=1,4 \ln s_{k}+1,6 \ln s_{H}-3 \ln (n+g)$. Luego, las elasticidades del producto con relación al ahorro en capital físico, capital humano y al término $(\mathrm{n}+\mathrm{g})$ son, respectivamente, 1,4; 1,6 y -3 .

b) En la ecuación (2.2):

$\ln y_{\text {Solow }}^{*}=0,54 \ln s_{k}-0,54 \ln (n+g)$. Por lo tanto, las elasticidades del producto con relación al ahorro en capital físico y al término $(\mathrm{n}+\mathrm{g})$ son 0,54 e $-0,54$, respectivamente.

Se observa que el modelo de capital humano (que trabaja con una concepción amplia de capital) presenta las elasticidades del producto con relación a sus determinantes, bastante superiores a las obtenidas mediante el modelo neoclásico de crecimiento. Esto significa que aquel modelo, al contrario de éste, es más adecuado para explicar el porqué de la diferencia de renta per capita en tiempo y espacio, lo que puede constastarse por medio del siguiente ejemplo propuesto por David Romer (1996:133-136), donde analiza la diferencia de producto por trabajador entre un país desarrollado (país 2) y otro en desarrollo (país 1), con base en la siguiente información:

Ambos países poseen iguales funciones de produción y tecnología; $\alpha=0,35$ y $\beta=0,40$; $s_{k 2}=2 s_{k 1}, s_{H 2}=2 s_{H 1} ; \mathrm{y}(\mathrm{n} 2+\mathrm{g})=0,8(\mathrm{n} 1+\mathrm{g})$. Con base en estos datos, tenemos que la diferencia en el producto por trabajador entre ellos es:

a) En el modelo neoclásico de crecimiento:

$y_{2}^{*}=1,6 y_{1}^{*}$. Luego, el producto por trabajador en el país desarrollado es solamente 1.6 veces mayor que en el país en desarrollo o emergente.

b) En el modelo de capital humano:

$y_{2}^{*}=15,62 y_{1}^{*}$. Así, el producto por trabajador de equilibrio de largo plazo del país desarrollado es casi 16 veces mayor que el del país en desarrollo o emergente. Este resultado muestra que las diferencias espaciales no extraordinarias en las tasas de ahorro y de crecimiento de la población explican de forma satisfactoria el contraste observado en el producto por trabajador entre unidades geográficas como países, regiones, microrregiones, estados y municipios. 
Podemos decir que los modelos que trabajan con una concepción amplia de capital consiguen explicar mejor las diferencias espaciales de rentas en estudios de corte transversal. Desde esta perspectiva, podemos incorporar al modelo de capital humano las variables de capital geográfico, de infraestructura e institucionales, y trabajar con una función de producción genérica del tipo: $Y_{t}=F\left(K_{t}, A_{t} L_{t}, H_{t}, G, I N F E S T_{t}, I N S T I T_{t}\right)$, donde $\mathrm{G}=$ capi- $^{-}$ tal geográfico (que es fijo y exógeno con relación al producto), INFEST = infraestructura e INSTIT $=$ variables institucionales.

El estudio empírico desarrollado en la cuarta sección de este trabajo se realiza tomando como base este tipo de función de producción, pero con los debidos ajustes a los datos disponibles para estimar las ecuaciones. Esto significa que se sigue de cerca la metodología adoptada en los trabajos de Carlos Azzoni et al. (2000), Fábio Torres y Jairo Méndez (2000) y Gerardo Esquivel (2000).

\section{Análisis descriptivo de las posibles causas de la concentración de la producción y desigualdad del producto per capita entre microrregiones del nordeste de Brasil}

Haremos ahora una breve exposición de las estadísticas básicas de las variables densidad económica (que mide la concentración geográfica de la producción en las microrregiones), educación (capital humano) y condiciones de vida de la población (potencial productivo de las personas, en una concepción amplia), donde se tiene por hipótesis que estas variables afectaron no sólo el nivel, sino también la tasa de crecimiento del producto per capita de las microrregiones del nordeste en el periodo 1970-1998. En el presente trabajo se optó por la siguiente división del nordeste: con base en un conjunto de 182 microrregiones (aglomerados de municipios con características geoeconómicas semejantes), se crearon los subconjuntos de la región metropolitana (MRM); del Litoral (MLIT); del Semi-árido (MSA), que engloba el Sertón, el Agreste, el São Francisco y el Parnaíba; del Cerrado (MCER); y de la Pre-amazonia (MPRM).

\section{Densidad económica: concentración geográfica}

de la producción microrregional

Según Paul Krugman (1991:13-34), la concentración geográfica de la produción en el nivel de país (o región) puede determinarse con base en los retornos crecientes, costos de transporte y demanda. O sea, si se admiten economías significativamente fuertes, la empresa típica deseará ofertar para el mercado nacional (o regional) a partir de una localidad única. Entre tanto, para minimizar los costos de transporte, la citada empresa escogerá una ubicación donde la demanda local sea importante. Sin embargo, ésta será grande en la 
localidad donde estén la mayoría de las empresas. Se observa, por lo tanto, que hay una cierta circularidad de influencia de los elementos que llevan a la concentración de la producción y, consecuentemente, a la formación de grandes mercados productores de bienes y servicios como, por ejemplo, los de las regiones metropolitanas de Fortaleza, Recife y Salvador.

La proximidad de grandes mercados también facilita la oferta de bienes intermediarios; torna más fácil la contratación de trabajadores de competencia técnica adecuada a las exigencias productivas de las empresas, y permite la difusión de información tecnológica entre las empresas de una misma industria. Podemos inferir, por lo tanto, que la concentración de la producción en una zona (o país), una vez establecida, presenta fuerzas endógenas capaces de mantenerla mientras se conserve la desigualdad representada por la siguiente ecuación: $\mathrm{F}>\frac{(1-\pi)}{2} x t$. Donde $\mathrm{F}$ son las economías de escala, $t$ es el costo unitario con transporte, $\pi$ es la participación que las manufacturas no vinculadas a los recursos naturales de localidades específicas que poseen en la economía de la región (o país) y $x$ son las ventas de una empresa manufacturera típica en una localidad determinada.

Analizando en forma sucinta el proceso de industrialización del nordeste con base en los fundamentos teóricos expuestos en los párrafos anteriores, se tiene:

Según Antônio Castro (1975;10-40), el objetivo de crear en esta región un centro autónomo de producción manufacturera no se alcanzó; y el resultado de las políticas de promoción de la industrialización del nordeste, en el periodo 1960-1970, fue el surgimiento de una industria moderna con baja vinculación anterior con el sector agropecuario, lo que significa un alto $\pi$; baja integración con la industria tradicional ya existente, que producía textiles y alimentos; y alta integración con la industria del centro-sur de Brasil, exportando bienes intermediarios que, en general, están marcados por fuertes economías de escala, o sea, F significativamente alto.

Ante lo expuesto, y considerando que en el inicio del proceso de industrialización del nordeste, 1960 a 1970, las regiones metropolitanas fueron privilegiadas en términos de inversiones productivas, se puede decir que se verificó la condición para la concentración productiva en esta región, o sea, $\mathrm{F}>\frac{(1-\pi)}{2} \quad x t$. Esto puede confirmarse con base en algunos datos sobre las microrregiones del nordeste en el periodo 1970-1998, (Francisco Rocha, 2003:44-60). En 1970, el subconjunto de las microrregiones metropolitanas (MRM), a pesar de representar apenas $1.3 \%$ del total, fue responsable de $37.7 \%$ del PIB total de las microrregiones y su densidad económica (producto por $\mathrm{km}^{2}$ ) es de us $\$ 471795$ (us $\$ 1 \mathrm{de}$ 1998). Por otro lado, los demás subconjuntos de microrregiones presentan una densidad económica de entre us\$ 5 000, MPRM, y us\$ 31 000, MLIT, aproximadamente.

La concentración de la producción en las MRM después de 1970 permanece significativamente alta, o sea, la participación de las MRM en el PIB total del conjunto de las microrregiones fue $40.4 \%$ en $1980,45,6 \%$ en 1990 y 43,5\% en 1998. Esto puede explicarse por 
las políticas de promoción de la industrialización en el nordeste, que privilegiaron inversiones en las regiones metropolitanas, como el complejo petroquímico de Camaçari (BahiaSalvador) que, instalado entre 1970 y 1977, inició actividades en 1978; el polo industrial portuario de Suape (Pernambuco-Recife), establecido a comienzos de la década de los setenta; y el III polo industrial del Nordeste (Ceará-Fortaleza), cuyo objetivo era estimular las actividades productivas de los sectores textil, de ropa, calzado y de tejidos, comenzó a instrumentarse en 1978. Estas inversiones, estimuladas por los incentivos crediticios y fiscales del gobierno federal, estimularon no sólo al sector manufacturero de las MRM, sino también a los de comercio y servicios. Hubo también en las MRM inversiones significativas del sector público en infraestrutura básica, como energía, saneamiento y habitación.

\section{Disponibilidad de capital humano}

En 1970, el capital humano de las MRM —-medido por el número de personas que, en la microrregión, poseen educación fundamental - era, como mínimo, nueve veces superior de las MLIT y, como máximo, 84 veces superior al de las MCER. En 1980, de las MRM era, como mínimo, ocho veces superior al de capital humano de las MLIT y, como máximo, 64 veces superior al de las MCER. Con respecto a la diferencia de calidad de capital humano en términos de personas que poseen enseñanza media o superior, se constata también que las MRM poseen una diferencia notable: como mínimo, ocho veces mejor.

\section{Condiciones de vida}

Tomando como base la clasificación del Programa de las Naciones Unidas para el Desarrollo (PNUD) para el índice de desarrollo humano (IDH), podemos clasificar los subconjuntos de microrregiones del nordeste de acuerdo con sus respectivos índices de condiciones de vida (ICV) en tres categorías:

$$
\begin{array}{ll}
0,0 \text { ICV 0,5 } & \text { Baja condición de vida } \\
0,5 \text { ICV 0,8 } & \text { Media condición de vida, y } \\
0,8 \text { ICV 1,0 } & \text { Alta condición de vida }
\end{array}
$$

Analizando la Tabla 1, se verifica que los subconjuntos de las MSA, MCER y MPRM, a pesar de mostrar cierto crecimiento en el ICV, presentan bajas condiciones de vida; el subconjunto de las MLiT pasa de una situación de baja condición de vida en el periodo 1970-1980 a una situación de media condición de vida en 1991; entre tanto, el subconjunto de las MRM la presenta a partir de 1980, lo que significa menores restricciones al potencial productivo de quienes viven en las regiones metropolitanas de Fortaleza, Recife y Salvador. 
Tabla 1

Índice de condición de vida

\begin{tabular}{lccccc}
\hline & MRM & MLIT & MSA & MCER & MPRM \\
\hline ICV70 & 0,4296 & 0,3446 & 0,3286 & 0,3283 & 0,3150 \\
ICV80 & 0,5406 & 0,4379 & 0,4101 & 0,4044 & 0,3815 \\
ICV91 & 0,6114 & 0,5079 & 0,4985 & 0,4924 & 0,4421 \\
\hline
\end{tabular}

Fuente: elaboración propia con base en las informaciones de los datos regionales de la Fundación Instituto Brasileño de Geografía y Estadística y Banco Mundial de 2001. ICV es el índice de condición de vida. En la tabla está expuesto el promedio de esta variable para cada subconjunto de microrregiones del nordeste.

\section{Diferencias en el nivel y en la tasa de crecimiento}

del producto per capita microrregional

En 1970, el promedio del PIB per capita de las MRM (US\$ 1 448) era aproximadamente el doble del de las MLIT (uS\$ 760), y el triple del de los demás subconjuntos, que presentaron un valor aproximado de us\$ 450 cada uno. En 1998, el promedio del PIB per capita de las MRM (US\$ 3 976) se superó por más del doble el de las MLIT (US\$ 1 772), y por casi cuatro veces el de los demás subconjuntos, que presentaron un valor aproximado de us\$ 1000 cada uno.

Con relación al crecimiento del PIB per capita, tenemos que: en el periodo 1970-1998, el promedio de las tasas anuales de crecimiento era de $3.6 \%$ para las MRM, 3.0\% para las MLIT, $3.0 \%$ para las MSA, $4.4 \%$ para las MCER y $1.5 \%$ para las MPRM. Por lo tanto, excepto por las MCER, los datos no proporcionan evidencias de reducción de la desigualdad de renta entre las microrregiones del nordeste.

Con base en las estadísticas básicas de las variables geográficas (distancia de los grandes mercados y concentración de la producción), de capital humano (número de personas que poseen educación fundamental) e institucional (condiciones de vida, que reflejan el potencial productivo de una persona y, en conjunto el potencial económico de una unidad geográfica), obtenemos las siguientes conclusiones preliminares sobre el porqué de las diferencias en el nivel y en la tasa de crecimiento del PIB per capita entre los subconjuntos de microrregiones del nordeste, si se admite que las variables citadas afectan tanto al nivel como a la tasa de crecimiento del producto microrregional.

Las MRM poseen el mayor PIB per capita porque reciben la influencia positiva de la proximidad de los grandes mercados y sus facilidades en términos de oferta de bienes intermediarios, mano de obra calificada y difusión de tecnología; tienen una mayor calidad de capital humano — como mínimo, ocho veces superior al de los demás subconjuntos de microrregiones - ; y presentan mejores condiciones de vida — renta familiar, salud, habitación, expectativa de vida al nacer, saneamiento-, lo que favorece el aprovechamiento del potencial productivo de las personas que viven en las regiones metropolitanas. 


\section{Datos y modelos empíricos utilizados en el estudio}

Los datos sobre PIB global de las microrregiones del nordeste del Brasil (182 en total) se obtuvieron de los censos agrícola, industrial, servicio y comercio, realizados por la Fundación Instituto Brasileño de Geografía y Estadística (FIBGE), durante varios años; y del valor adicionado fiscal de los estados, también efectuado durante varios años. El PIB per capita se obtuvo mediante la división del PIB global entre la población de la microrregión.

Los trabajos que analizan el proceso de crecimiento económico suelen utilizar como proxy del capital humano la tasa de matrícula en la enseñanza fundamental y media. Robert Barro (1991:408-422) critica este proceder y considera que la tasa de matrícula corresponde al flujo de inversión en capital humano y no a la calidad del mismo, existente en una unidad geográfica. Para resolver tal problema, el autor citado sugiere que se utilicen como proxy valores corrientes y desfasados de las tasas de matrículas en las enseñanza fundamental y media. En el presente trabajo se optó por usar el número de personas con los anteriores grados y superior, lo que corresponde, de forma más fidedigna, a la calidad de capital humano existente en las microrregiones del nordeste. Las regresiones de corte transversal, para el nivel del PIB per capita, se estiman usando el número de individuos con educación fundamental, media o superior en las microrregiones durante el año inicial (para evitar problema de endogeneidad) como variable explicativa — al lado de las variables geográfica e institucional-, cuando se espera que la señal de la variable en cuestión sea positiva. O sea, cuanto mayor sea la calidad de capital humano, mayor será el nivel y la tasa de crecimiento del PIB per capita.

Se utiliza el promedio de distancia de las microrregiones hasta el sitio principal de producción y comercialización de bienes y servicios del nordeste (Fortaleza-RecifeSalvador) como proxy de la variable geográfica. Se optó por ella porque, como se vió anteriormente, la distancia de los grandes mercados y sus facilidades de oferta de bienes intermediarios, mano de obra y difusión de tecnología tienen efectos significativos sobre la concentración de la produción en determinadas localidades. Por lo tanto, las regresiones de corte transversal, para el nivel del PIB per capita, se estiman usando el promedio de distancia de las microrregiones hasta el principal punto de producción y comercialización de bienes y servicios como variable explicativa — al lado de las variables de capital humano e institucional—, donde se espera que la señal de la misma sea negativa. Es decir, cuanto mayor sea la distancia, menor serán el nivel y la tasa de crecimiento del PIB per capita.

Se utiliza el índice de condiciones de vida (ICv) como proxy de la variable institucional porque se construye a partir de 18 indicadores que dependen, en gran medida de decisiones políticas e institucionales. O sea, el ICV está constituido por los siguientes indicadores, fuera de la renta familiar per capita y de la tasa de analfabetismo: porcentaje de niños que no frecuentan la escuela; desfase escolar de los niños; porcentaje de los niños que trabajan; 
condiciones de las viviendas; porcentaje de la población que tiene acceso al agua y alcantarillas (estas dos variables reflejan, en parte, la cuestión de la infraestructura de las microrregiones); esperanza de vida al nacer; y mortalidad infantil.

Como se vió en la tercera sección, este índice puede dividirse en las siguientes categorias:

$\begin{array}{ll}0,0 \text { ICV 0,5 } & \text { Baja condición de vida; } \\ 0,5 \text { ICV 0,8 } & \text { Media condición de vida; y } \\ 0,8 \text { ICV 1,0 } & \text { Alta condición de vida. }\end{array}$

Tenemos por hipótesis que las condiciones de vida (ICV) son afectadas por el crecimiento económico, mas éste no es sustentable a largo plazo sin decisiones políticas e institucionales capaces de mejorar las condiciones de vida de la población. Así, tenemos que el crecimiento económico afecta y es afectado por las condiciones de vida. El efecto de las condiciones de vida (ICV) sobre el nivel y la tasa de crecimiento del producto es resultado de las restricciones sobre el potencial productivo de las personas que viven en una determinada localidad. Cuanto menor sea el ICV de una localidad - lo que indica alto porcentaje de niños trabajando, baja calidad de las viviendas, bajo porcentaje de familias con agua y alcantarillas, alta tasa de mortalidad infantil y baja expectativa de vida al nacer-, menor será el potencial productivo de sus habitantes, lo que resulta, en términos macroeconómicos, en bajo nivel y tasa de crecimento del producto per capita de esa localidad.

Las regresiones de corte transversal, para el nivel del PIB per capita, se estiman usando el ICV en el año inicial, para evitar problema de endogeneidad, como variable explicativa —al lado de las variables de capital humano y geográfica — , donde se espera que la señal de la variable en cuestión sea positiva. O sea, cuanto mayor sea el ICV (menores restricciones sobre el potencial productivo de las personas residentes en una localidad), mayor será el nivel y la tasa de crecimiento del PIB per capita.

\section{Modelos empíricos: estimación de las ecuaciones de corte transversal, metodología de la descomposición del diferencial de renta y coeficiente de Gini}

El estudio empírico se desarrolla mediante los siguientes pasos:

Paso 1: se estima una regresión de corte transversal de microrregiones del nordeste, tomando como variable dependiente el PIB per capita de las microrregiones en 1998, y como variables explicativas las geográficas, que son exógenas con relación al PIB, y las de capital humano e institucional. Se utiliza el valor en el tiempo inicial de esas dos últimas para evitar el problema de endogeneidad. Los resultados del modelo estimado mostrarán el efecto de cada una de las variables explicativas sobre el PIB per capita de las microrregiones. 
Paso 2: con base en los coeficientes estimados de la regresión utilizada en el Paso 1, se analiza la participación de las variables geográfica, de capital humano e institucional en la diferencia del promedio de renta per capita entre los subconjuntos de microrregiones del nordeste: región metropolitana (MRM), Litoral (MLIT), Semi-árido (MSA), Cerrado (MCER) y Pre-amazonia (MPRM), tomando como base la siguiente metodología de descomposición del diferencial de renta: primero, se establece con base en los conjuntos definidos de microrregiones —MRM, MLIT, MSA, MCER y MPRM - cuáles se compararán para establecer la diferencia en el nivel promedio del PIB per capita. Por ejemplo, se puede comparar la re-gión metropolitana (MRM) con las demás que componen el nordeste; segundo, calcular cuánto contribuye cada variable explicativa (geográfica, capital humano e institucional) a la diferencia en el promedio de nivel del PIB per capita entre dos subconjuntos de microrregiones, por ejemplo, MRM y MLIT, con base en la siguiente ecuación:

$$
\bar{Y}_{M R M}-\bar{Y}_{M L I T}=\Sigma_{i=1}^{k} \hat{\beta}_{i}\left(\bar{X}_{M R M, i}-\bar{X}_{M L I T, i}\right)
$$

donde:

$\overline{\mathrm{Y}}_{\text {MRM }} \quad$ PIB per capita medio del subconjunto de microrregiones de la región metropolitana (MRM);

$\overline{\mathrm{Y}}_{\text {мLтт }}$ PIB per capita medio del subconjunto de microrregiones del Litoral (MLIT);

$\hat{\beta}_{i} \quad$ Coeficiente estimado, en el Paso 1, de la variable explicativa $i$, de la regresión del PIB per capita, en 1998, en función de las variables geográfica, de capital humano e institucional;

$\overline{\mathrm{X}}_{\text {MRM }} \quad$ Promedio del valor de la variable explicativa $i$ en el subconjunto de microrregiones MRM;

$\overline{\mathrm{X}}_{\text {мцтт }} \quad$ Promedio del valor de la variable explicativa $i$ en el subconjunto de microrregiones MLIT; y

$\hat{\beta}_{\mathrm{i}}\left(\overline{\mathrm{X}}_{\mathrm{MRM}, \mathrm{i}}-\overline{\mathrm{X}}_{\mathrm{MLLT,}}\right)$ impacto de la variable explicativa $i$ en la diferencia de PIB per capita entre los subconjuntos de microrregiones MRM (región metropolitana) y MLIT (Litoral).

Paso 3: se estudian, a través del coeficiente de desigualdad de Gini, los efectos aislados y conjuntos de las variables geográficas, de capital humano e institucional sobre la desigualdad de renta entre microrregiones del nordeste. Este estudio se desarrolla de la siguiente forma: primero, se calcula el coeficiente de desigualdad de renta per capita de Gini original para las 182 microrregiones, usando los datos del PIB per capita de 1998. Segundo, se estima una nueva renta per capita para cada microrregión, $\mathrm{Y}_{\mathrm{Nj}}$, con base en la siguiente ecuación: 


$$
Y_{N j}=Y_{j}-\hat{\beta}_{i}\left(X_{i, j}-\bar{X}_{i, .}\right)
$$

Esta ecuación determina la nueva renta per capita (PIB per capita) de la microrregión $j$, en 1998; después se considera el efecto aislado de la variable explicativa $i$, donde,

$\mathrm{Y}_{\mathrm{Nj}}$ nueva renta per capita de la microrregión $j$;

$\mathrm{Y}_{\mathrm{j}} \quad$ renta per capita original de la microrregión $j$;

$\hat{\beta_{i}}$ coeficiente estimado de la variable explicativa $i$, en la regresión de la renta per capita, en 1998, en función de las variables geográfica, de capital humano e institucional, modelo del Paso 1;

$\mathrm{X}_{\mathrm{i}, \mathrm{j}}$ valor de la variable explicativa $i$ en la microrregión $j$;

$\mathrm{X}_{\mathrm{i}, \text {. }} \quad$ valor medio de la variable explicativa $i$ llevándose en consideración todas las microrregiones.

Tercero, con base en los 182 valores de la nueva renta per capita estimada, $\mathrm{Y}_{\mathrm{Nj}}$, se calcula el nuevo coeficiente de desigualdad de Gini. Se puede deducir el efecto aislado de la variable explicativa $i$ sobre la desigualdad de renta per capita entre las microrregiones mediante la comparación de este coeficiente con el original. Por último, se debe repetir ese procedimiento con cada una de las variables explicativas para definir el efecto aislado de cada una sobre la desigualdad de renta per capita entre las microrregiones.

\section{Resultados empíricos}

Producto interno bruto per capita y las variables

de capital humano, geográfica e institucional

Se hace, ahora, un análisis econométrico de la relación entre el PIB per capita de las microrregiones en 1998 y las variables geográfica, de capital humano e institucional. Se utiliza el valor en el tiempo inicial de esas dos últimas, para evitar el problema de endogeneidad. Los resultados del modelo estimado mostrarán el efecto de cada una de las variables explicativas sobre el PIB per capita de las microrregiones. Se debe observar que éste es una extensión del modelo de crecimiento de capital humano propuesto por Gregory Mankiw, David Romer y David Weil (1992). Los criterios de aceptación de todos los modelos presentados en este trabajo se basan en las siguientes estadísticas de prueba, y sus respectivas probabilidades: t-student, $\mathrm{F}, \mathrm{y} \mathrm{R}^{2}$ ajustado.

En la Tabla 2 se observa que todas las covariables presentan la señal esperada y son estadísticamente significativas a 5\%, tanto individual como colectivamente. Se verifica también que para varias especificaciones de capital humano, a medida que se introducen las variables geográfica e institucional, el grado de ajuste del modelo mejora significativamente, o 


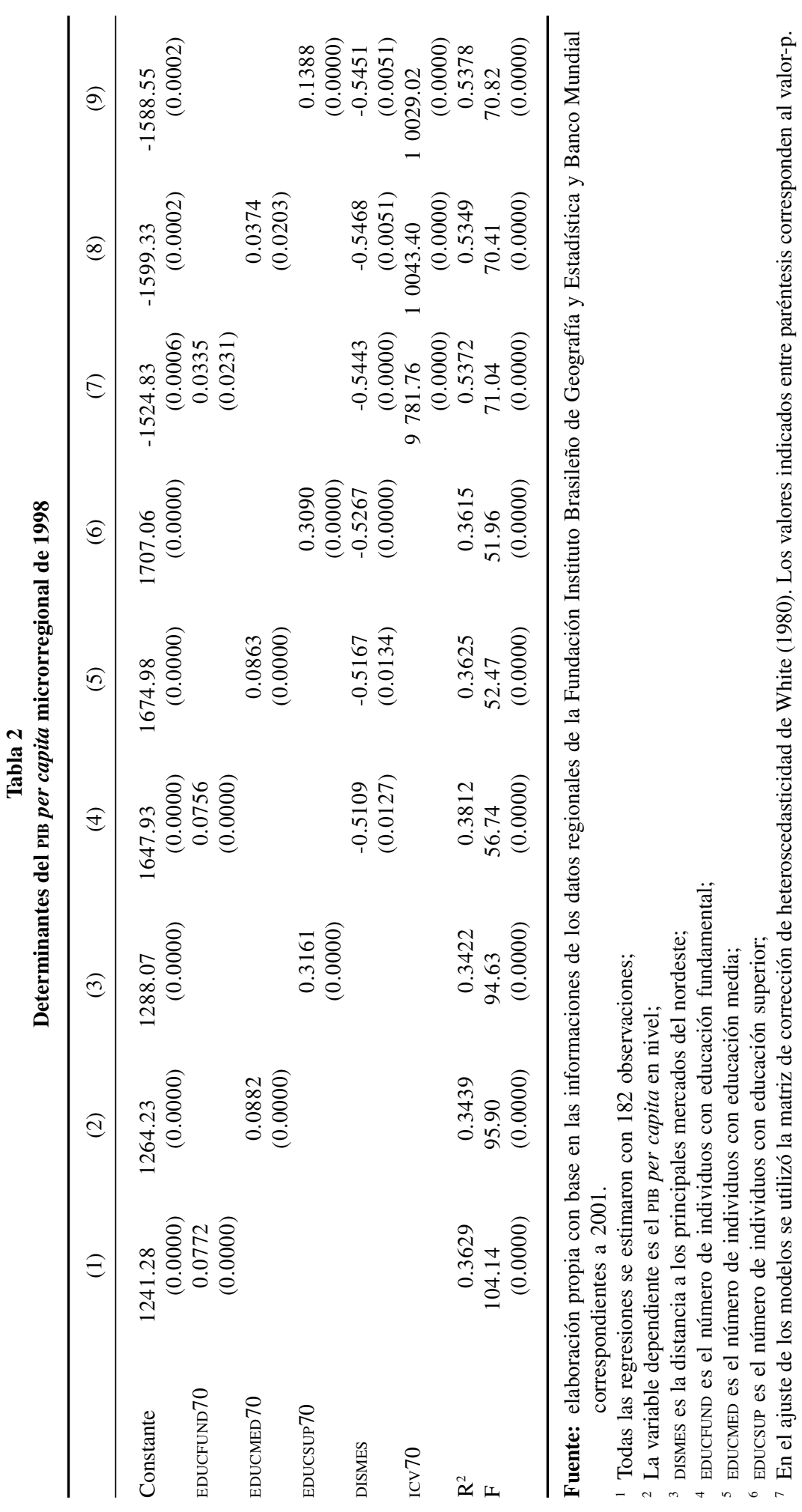


sea, el $\mathrm{R}^{2}$ ajustado pasa de $36 \%$ (modelo que tiene como variable explicativa solamente el capital humano) a 53\% (modelo con todas las covariables). Esto significa que todas las variables explicativas son relevantes para explicar el PIB per capita microrregional de 1998, lo que significa que el modelo teórico propuesto es adecuado.

Con base en las evidencias econométricas, que respaldan el modelo teórico que fundamenta el presente trabajo, se puede decir que: $a$ ) la variable capital humano influye positivamente en el producto microrregional, pero aisladamente no consigue explicar totalmente el nivel del producto microrregional en un año determinado, lo que exige una extensión del modelo; $b$ ) la variable geográfica tiene una relación negativa con el producto. El promedio de distancia entre las microrregiones y el principal eje de producción y comercialización de bienes y servicios del nordeste (Fortaleza-Recife-Salvador) se relaciona negativamente con el PIB per capita microrregional. Esto significa que cuanto mayor sea la distancia a los grandes mercados y sus facilidades de oferta de bienes intermediarios, mano de obra calificada y difusión tecnológica, menor será el producto per capita microrregional; c) y, por último, las condiciones de vida de las personas de una microrregión tienen relación positiva con el producto per capita de la misma, esto es, cuanto mejores sean las condiciones de vida de las personas de una microrregión (medidas de acuerdo con el ICv), mayor será el potencial productivo de esas personas. En términos macroeconómicos, esto incrementará las posibilidades económicas de la microrregión, y nivel y tasa de crecimiento del producto per capita.

\section{Metodología de la descomposición del diferencial de renta}

En 1998, las MRM tenían un producto per capita medio de uS\$ 3 976, mientras las MLIT, MSA, MCER Y MPRM tenían promedios de uS\$ 1 772, uS\$ 1 134, uS\$ 1395 y us\$ 849, respectivamente. Esto muestra que existe una desigualdad de producto per capita (o renta) muy grande entre el subconjunto de las MRM y los demás de microrregiones del nordeste. El objetivo de la presente sección es analizar las causas de esa diferencia.

En el apartado anterior se vió que los subconjuntos de microrregiones poseían diferencias significativas, favorables para las MRM, en términos de las variables de capital humano, geográfica e institucional, lo que llevó a la hipótesis de que son estas diferencias las que provocan que las MRM presenten un promedio del producto per capita superior al de los productos per capita de los demás subconjuntos. Se asumió, por lo tanto, que las variables de capital humano, geográfica e institucional tenían una influencia significativa sobre el nivel del producto per capita en 1998. Esta hipótesis fue confirmada empíricamente, mediante los resultados de las regresiones de corte-transversal.

Esto permite analizar los efectos de las variables de capital humano, geográfica e institucional sobre la diferencia de producto per capita entre los subconjuntos de microrre- 
giones del nordeste, tomándose por base la metodología de la descomposición del diferencial de renta.

La metodología de la descomposición del diferencial de renta o producto per capita —bastante conocida y utilizada en trabajos significativos como Carlos Azzoni et al. (2000), Fábio Torres y Jairo Méndez, (2000) y Gerardo Esquivel (2000) - consiste en descomponer las diferencias de producto per capita microrregional de acuerdo con las principales fuentes, o variables, generadoras de esas diferencias. Para llevar adelante este ejercicio es ne-cesario dividir el conjunto total de microrregiones del nordeste de Brasil en subconjuntos con características específicas. Se adopta aquí la misma división presentada en la tercera sección, o sea, región metropolitana (MRM), Litoral (MLIT), Semi-árido (MSA), Cerrado (MCER) y Pre-amazónicas (MPRM). En segundo lugar, se calculan las contribuciones de las variables de capital humano (EDUCFUND), geográfica (DISMES) e institucional (ICV) a la diferencia del producto per capita entre dos subconjuntos de microrregiones, por ejemplo, MRM versus MLIT, de acuerdo con la ecuación 4.1, expuesta anteriormente:

$$
\bar{Y}_{M R M}-\bar{Y}_{M L I T}=\Sigma_{i=1}^{k} \hat{\beta}_{i}\left(\bar{X}_{M R M, i}-\bar{X}_{M L I T, i}\right)
$$

Se utilizan en este análisis de descomposición los coeficientes estimados en la ecuación 7 , Tabla 2, de las variables de capital humano (EDUCFUND), geográfica (DISMES) e institucional (ICV), pues dicha ecuación incluyó todas las variables explicativas relevantes para la determinación del producto per capita en nivel en 1998. Los resultados obtenidos se encuentran en la Tabla 3, donde el primer subconjunto de microrregiones de cada par es el de las MRM (microrregiones relativamente más ricas), lo que lleva a un ejercicio de descomposición de las MRM versus los demás subconjuntos.

Analizando en forma pormenorizada los números expuestos en la Tabla 3, se obtienen algunos resultados notables. Primero, la diferencia del producto per capita explicada por la variable de capital humano varía de $24 \%$ entre las MRM y las MPRM, a $32 \%$ entre las MRM y las mLIT. Segundo, la diferencia explicada por la variable institucional varía de $38 \%$ entre las MRM y las MSA, a $42 \%$ entre las MRM y las MCER. Tercero, la diferencia explicada por la variable geográfica varía de $2 \%$ entre las MRM y las MSA, a 17\% entre las MRM y las MCER.

Ante lo expuesto, podemos afirmar que las variables de capital humano e institucional son las principales responsables de las diferencias de producto per capita o renta entre el subconjunto de microrregiones más ricas, esto es, las MRM, y los demás subconjuntos de microrregiones del nordeste. Esta es una constatación bastante importante, pues las variables de capital humano, representadas por la educación fundamental e institucional, representada por el índice de condiciones de vida, son de política económica. Esto significa que está en manos de los formuladores de políticas públicas del nordeste, y de Brasil, elaborar estrategias que lleven un aumento de la calidad de capital humano y mejoren las 
Tabla 3

Descomposición del diferencial de producto per capita microrregional

\begin{tabular}{lcccc}
\hline & MRM $v s$ MLIT & MRM $v s$ MSA & MRM $v s$ MCER & MRM $v s$ MPRM \\
\hline $\begin{array}{l}\text { Var. capital humano } \\
\text { (EDUCFUND) }\end{array}$ & 32 & 26 & 29 & 24 \\
$\begin{array}{l}\text { Var. Geográfica } \\
\text { (DISMES) }\end{array}$ & 4 & 2 & 17 & 12 \\
Var. Institucional & 40 & 38 & 42 & 38 \\
(ICV) & 76 & 66 & 88 & 74 \\
Total Explicado & 24 & 34 & 12 & 26 \\
Residuo & & & & \\
\hline
\end{tabular}

Fuente: elaboración propia con base en las informaciones de los datos regionales de la Fundación Instituto Brasileño de Geografía y Estadística y Banco Mundial correspondientes a 2001.

Nota: Los números en el cuerpo de la tabla corresponden a la participación percentual de cada covariable el diferencial de producto per capita entre los dos subconjuntos de microrregiones que están siendo comparados.

condiciones de vida de las personas que habitan en las microrregiones relativamente pobres del nordeste.

Solamente políticas públicas de esa naturaleza pueden mejorar el potencial productivo de quienes viven en microrregiones pobres, lo que, a su vez, lleva a un aumento del potencial económico de estas microrregiones, resultando tanto en mayor nivel como una alta tasa de crecimiento del producto per capita. Esto posiblemente llevará no sólo a una reducción de la diferencia de producto per capita entre el subconjunto de microrregiones relativamente más ricas y los demás subconjuntos, sino también a una reducción de la pobreza absoluta en la región nordeste. Como se sabe, la pobreza se reduce por el camino del crecimiento económico y de una mejor distribución de renta. Sobre este último punto se analizará en la próxima sección la influencia de las variables de capital humano, geográfica e institucional sobre la concentración de renta en las microrregiones del nordeste, tomando como base la metodología del índice de concentración de renta de Gini, original y estimado.

\section{Efecto de las variables de capital humano, geográfica \\ e institucional sobre la concentración de renta \\ de las microrregiones del nordeste}

En esta sección se analizan los efectos de las variables de capital humano, geográfica e institucional sobre el índice de concentración de renta de Gini de las microrregiones del nordeste. Para el cálculo del índice de Gini, original y estimado, se supone igualdad de renta dentro de cada microrregión. O sea, se admite que todas las personas residentes en una microrregión tiene la misma renta microrregional media. De esa forma, la medida de 
concentración que se utiliza en el presente trabajo puede considerarse como un coeficiente de Gini establecido con base en la población de cada microrregión del nordeste (Gerardo Esquivel, 2000:40-41). Los efectos aislados y en conjunto de las variables de capital humano, geográfica e institucional sobre la concentración de renta de las microrregiones pueden medirse de la siguiente forma: primero, se calcula el índice Gini original para las 182 microrregiones del nordeste usando el PIB per capita observado en 1998; segundo, se estima la nueva renta per capita, $\mathrm{Y}_{\mathrm{Nj}}$, para cada microrregión con base en la ecuación 4.2, (expuesta en la página 174):

$$
Y_{N j}=Y_{j}-\hat{\beta}_{i}\left(X_{i, j}-\bar{X}_{i,}\right)
$$

Esta ecuación determina la nueva renta per capita (PIB per capita) de la microrregión $j$, en 1998; después, se considera el efecto aislado de la variable explicativa $i$, donde,

$\mathrm{Y}_{\mathrm{Nj}}$ es la nueva renta per capita de la microrregión $j$;

$\mathrm{Y}_{\mathrm{j}}$ es la renta per capita original de la microrregión $j$;

$\hat{\beta}_{\mathrm{i}}$ es el coeficiente estimado de la variable explicativa $i$, en la regresión de la renta per capita, en 1998, con base en las variables geográfica, de capital humano e institucional, ecuación 7 de la Tabla 2;

$\mathrm{X}_{\mathrm{i}, \mathrm{j}}$ es el valor de la variable explicativa $i$ en la microrregión $j$;

$\overline{\mathrm{X}}_{\mathrm{i}, \text {. }}$ es el valor medio de la variable explicativa $i$ llevándose en consideración todas las microrregiones.

Tercero, con base en los 182 valores de la nueva renta per capita estimada, $\mathrm{Y}_{\mathrm{Nj}}$, se calcula el nuevo coeficiente de concentración de Gini. Comparándolo con el coeficiente original puede medirse el efecto aislado de la variable explicativa $i$ sobre la concentración de renta per capita entre las microrregiones. Se debe repetir ese procedimiento para cada una de las variables explicativas, con el fin de determinar el efecto de cada una sobre la desigualdad de renta per capita entre las microrregiones.

Debe evitarse comparar el índice de Gini calculado con base en la población de unidades geográficas como estado o microrregión, con el determinado a partir de microdatos. En el cálculo de este último se considera la diferencia de renta dentro de cada unidad geográfica. El siguiente ejemplo ayuda a esclarecer por qué debe hacerse esta distinción: Gerardo Esquivel (2000:42), trabajando con la metodología del índice de Gini estimado a partir de la población de los estados de México, obtuvo un índice de 0.282, para 1995. Sin embargo, el Banco Interamericano de Desarrollo, usando microdatos, presentó un índice de Gini para México de 0.582, para 1996. 
La Tabla 4 muestra los índices de Gini originales y estimados, calculados mediante el programa POVCAL, desarrollado por Schaochua Chen, del Banco Mundial. El número de la primera columna representa el índice de Gini original, obtenido a partir de los datos observados del producto per capita de las microrregiones. En la segunda columna se tiene el índice estimado al controlarse el efecto de la variable de capital humano. Obsérvase, por lo tanto, que la inclusión de esta variable (educación fundamental) reduce la concentración de renta en las microrregiones en $7.3 \%$. O sea, en el caso de que no hubiese una diferencia tan grande en términos de educación entre las microrregiones, se tendría una menor concentración de renta en las mismas.

Tabla 4

Índice de Gini de las microrregiones, 1998

\begin{tabular}{cccc}
\hline GINIORIG & GINIH & GINIHD & GINIHDI \\
\hline 0.41 & 0.38 & 0.32 & 0.26 \\
\hline
\end{tabular}

Fuente: elaboración propia con base en las informaciones de los datos regionales de la Fundación Instituto Brasileño de Geografía y Estadística y Banco Mundial correspondientes a 2001.

La tercera columna muestra el índice de Gini al incluir en el análisis, además de la variable de capital humano, la geográfica (distancia al eje principal de producción y comercialización de bienes y servicios del nordeste). Se verifica, por otro lado, que esta inclusión reduce la concentración de renta en $16 \%$, si se le compara con el índice de Gini estimado de la segunda columna, y 22\%, comparada con el índice original de la primera columna. Luego, la proximidad de los grandes mercados y sus facilidades en términos de insumos, mano de obra y tecnología tiene un papel importante en la explicación de la concentración de renta microrregional. La cuarta columna expone el índice de Gini estimado al incluir en el análisis, además de las variables de capital humano y geográfica, la institucional (índice de condiciones de vida, que proporciona una idea del potencial productivo de las personas $y$, consecuentemente, de la economía de una microrregión). Se observa que la concentración de renta entre las microrregiones se reduce en $18.7 \%$, comparada con el índice de Gini estimado de la tercera columna, y en $36.6 \%$, contra el índice de Gini original de la primera columna.

Se concluye que si no hubiese diferencias significativas en las variables de capital humano, geográfica e institucional entre las microrregiones del nordeste, la concentración de renta entre ellas sería significativamente menor. Por lo tanto, considerando que las variables de capital humano e institucional son de políticas públicas y que pueden mejorarse, está en las manos de los formuladores de políticas y de los gobernantes del nordeste, y de Brasil, buena parte de la tarea de reducir la concentración de renta y, consecuente- 
mente, la pobreza en esta región. Solamente políticas públicas que busquen aumentar el potencial productivo de las personas que viven en el Nordeste lograrán estimular el crecimiento económico con equidad social en esta región.

\section{Conclusión}

En este trabajo se tuvo por objetivo analizar empíricamente las cuestiones relacionadas a la desigualdad de renta per capita entre microrregiones y subconjuntos de microrregiones (región metropolitana, Litoral, Semi-árido, Cerrado Pre-amazónica) del nordeste, en el periodo 1970-1998, destacando el papel de las políticas públicas. Por lo tanto, se analizaron: a) los efectos de las variables geográficas, de capital humano e institucional sobre el nivel del producto per capita de las microrregiones del nordeste en 1998; b) la participación de las variables geográfica, de capital humano e institucional en la magnitud de la diferencia de renta per capita media entre los subconjuntos de microrregiones; y c) cómo las variables geográfica, de capital humano e institucional afectan de forma aislada y en conjunto la concentración de renta entre las microrregiones del Nordeste.

Las evidencias econométricas, que respaldaron el modelo teórico que fundamentó el presente trabajo, mostraron que: $a$ ) la variable capital humano influye positivamente en el producto microrregional, pero aisladamente no consigue explicar el nivel de este producto en un año específico, lo que exige una extensión del modelo; $b$ ) la variable geográfica posee una relación negativa con el producto: la distancia media de las microrregiones al principal eje de produción y comercialización de bienes y servicios del nordeste (FortalezaRecife-Salvador) está negativamente relacionada con el PIB per capita microrregional. Esto significa que cuanto mayor sea la distancia a los grandes mercados y sus facilidades de oferta de bienes intermediarios, mano de obra calificada y difusión tecnológica, menor será el produto per capita microrregional; y c) las condiciones de vida de las personas de una microrregión tienen relación positiva con el producto per capita de la misma, esto es, cuanto mejores son las condiciones de vida, mayor es el potencial productivo de esas personas. En términos macroeconómicos, esto resultará en un incremento del potencial económico de la microrregión, y del nivel y tasa de crecimento del producto per capita de la misma.

El ejercicio de descomposición del diferencial del producto per capita o renta obtuvo resultados notables. Primero, la parte de la diferencia del producto per capita explicada por la variable de capital humano varió de $24 \%$ entre las MRM y las MPRM, a $32 \%$ entre las MRM y las MLIT. Segundo, explicada por la variable institucional varió de $38 \%$ entre las MRM y las MAS, y $42 \%$ entre las MRM y las MCER. Tercero, explicada por la variable geográfica varió de $2 \%$ entre las MRM y las MAS, a 17\% entre las MRM y las MCER. 
Podemos afirmar que las variables de capital humano e institucional son las principales responsables de las diferencias de producto per capita o renta entre el subconjunto de microrregiones más ricas, esto es, las MRM, en contraposición a los demás subconjuntos de microrregiones del Nordeste.

El análisis de los efectos de las variables de capital humano, geográfica e institucional sobre el índice de concentración de renta de Gini mostró que si no hubiese diferencias significativas en estas variables entre las microrregiones del Nordeste, la concentración de renta entre ellas sería al menos $37 \%$ menor. Por lo tanto, considerando que las variables de capital humano e institucional son variables de políticas públicas y que pueden mejorarse - mediante el aumento en el número de personas con educación básica, con la reducción de la mortalidad infantil, la erradicación del trabajo infantil, el aumento del número de personas con acceso a los servicios de agua y alcantarillas, y crear condiciones dignas de vivienda-, está en las manos de los formuladores de políticas y de los gobernantes del Nordeste, buena parte de la tarea por reducir la concentración de renta y, consecuentemente, la pobreza en esta región. Es necesario el desarrollo de políticas públicas que busquen aumentar el potencial productivo de las personas que viven en él para lograr que se estimule el crecimento económico con equidad social en esta zona. Mientras las políticas públicas no tengan en cuenta a las personas, continuarán existiendo el Nordeste de los más ricos, que viven en las regiones metropolitanas, y el de los pobres, que habitan en el Sertón.

\section{Bibliografía}

Azzoni, Carlos Roberto, Naércio Menezes Filho, Tatiane Menezes, Raul Silveira Neto, "Geography and income convergence among Brazilian states", Inter-American Development Bank, Research Department, Working Paper 395, United States, 2000.

Barro, Robert, "Economic growth in a cross-section of countries", Quarterly Journal of Economics, vol. 106, 1991, United States, pp. 407-443.

y Xavier Sala - I - Martin, Economic growth, United States, McGraw-Hill, INC, 1995.

Castro, Antônio Barros, 7 ensaios sobre a economia brasileira, Brasil, FORENSE, 1975.

Esquivel, Gerardo, "Geografia y Desarrolo Económico en México", Inter-American Development Bank, Research Department, Working Paper 389, United States, 2000.

Fundación Instituto Brasileño de Geografía y Estadística e Banco Mundial, Dados Regionais, Disponível em: http://www.ipea.gov.br, Acesso em: 21 jan. 2001.
Furtado, Celso, Formação econômica do Brasil, Brasil, Nacional, 1976.

Gomex, Gustavo Maia, José Raimundo Oliveira Vergolino, A macroeconomia do desenvolvimento nordestino: 1960/1994, Prêmio Pernambucano de Economia Dirceu Pessoa - 94, IEPE, Brasil, 1995.

Grupo de Trabalho para o Desenvolvimento do Nordeste, Uma política de desenvolvimento econômico para o Nordeste, SUDENE, Brasil, 1967.

Krugman, Paul, Geography and Trade, MITPress, United States, 1991.

Mankiw, N. Gregory, David Romer y David Weil, "A contribution to the empirics of economic growth", NBER, WP, núm. 3541, United States, 1992.

Morales, Rolando, Ervin Galoppo, Luis Carlos Jemio, Maria Carmem Choque y Natacha Morales, "Bolivia: geografía y desarrolo económico", InterAmerican Development Bank, Research Department, Working Paper 387, United States, 2000.

Romer, Paul, "Increasing returns and long run growth", JPE, vol. 94, núm. 1002-1037, United States, 1986. 
Romer, David, Advanced Macroeconomics, United States, McGraw Hill, 1996.

Solow, Robert, "A contribution to the theory of economic growth", Quarterly Journal of Economics, vol. 70, United States, 1956, pp. 65-94.

Torres, Fábio Sánches y Jairo Núñez Méndez, "Geography and economic development in Colombia: a municipal approach", Inter-American Development Bank, Research Department, Working Paper 408, United States, 2000.

Vergolino, José Raimundo Oliveira y Aristides Monteiro Neto, "A hipótese de convergência da renda: um teste para o Nordeste do Brasil com dados microrregionais, 1970-1993", Revista Econômica, Nordeste, vol. 27, núm. 4, Brasil, 1996, pp. 701-724.

Programa das Nações Unidas para o Desenvolvimento, Atlas de Desenvolvimento Humano do Brasil, Fundação João Pinheiro, 1998

Rocha, Francisco José Sales, "Crescimento, Desigualdade e Concentração de Renda:Uma Análise Empírica a Partir das Microrregiões do Nordeste do Brasil - 1970/1998”, tesis de doctorado, PIMEs, Universidade Federal de Pernambuco, Brasil, 2003. 